\title{
Supplement to "Pervasive diffusion of climate signals recorded in ice-vein ionic impurities" by Felix S. L. Ng
}

5 Movies S1-S5: Here, captions only. Access the movies via doi:10.15131/shef.data.12739169. Please use https://figshare.com/s/aa059ab52b73f472f3fd during the review stage.

Figs. S1-S2

10 Movie S1: Modelled evolution of a signal peak in our GRIP control run (top panels) and an otherwise identical run where the Gibbs-Thomson effect is turned off by setting $\gamma=0$ (bottom panels), from 0.5 to $130 \mathrm{kyr}$. The horizontal coordinate $z^{\prime}$ measures displacement in the material reference frame. Near the top left, $t$ denotes the age of the ice at $z^{\prime}=0$, and $z$ its depth below the surface; $t$ and $z$ apply to the movie frames in both runs. The simulated variables are bulk solute concentration $c_{\mathrm{B}}$ (left), vein solute concentration $c$ (middle), anomalous velocity $w_{\mathrm{c}}$, porosity $\phi$ and vein curvature $r_{v}$ (right). Grey curves in the left-hand

15 panels indicate the initial doped peak. Snapshots from the movie are shown in Fig. 4.

Movie S2: Modelled evolution of a signal peak in our EPICA control run (top panels) and an otherwise identical run where the Gibbs-Thomson effect is turned off by setting $\gamma=0$ (bottom panels), from 4 to $460 \mathrm{kyr}$. The panels are as organised in Movie S1. Snapshots from the movie are shown in Fig. 5.

Movie S3: Modelled evolution of two neighbouring peaks in a GRIP run from 0.5 to $70 \mathrm{kyr}$, using the control parameters of

20 the run in Fig. 4a and Movie S1 (top panels). $t$ denotes the age of the ice at $z^{\prime}=0$, and $z$ its depth below the surface. Diffusional spreading causes the peaks to merge as they approach each other under vertical compression. Snapshots from the movie are shown in Fig. 9.

Movie S4: Simulated evolution of a 20-m long sequence of $c_{\mathrm{B}}$ signals at the GRIP site from 0.5 to $100 \mathrm{kyr}$, in three experiments assuming the molecular diffusivities $D$ (Table 1), $0.1 D$ and 0.03D. $t$ denotes the age of the ice at $z^{\prime}=0$, and $z$ its depth below

25 the surface. The initial doped signal is made from the superposition of 300 random Gaussian peaks at decimetre scale. The upper panel shows the whole signal sequence as it evolves, resizing the axes occasionally to expand upon details. The lower panel focusses on the stretch $z^{\prime}=-2$ to $2 \mathrm{~m}$. 
Movie S5: Simulated evolution of a 80-m long sequence of $c_{\mathrm{B}}$ signals at the EPICA site from 5 to $500 \mathrm{kyr}$, in three experiments assuming the molecular diffusivities $D$ (Table 1), $0.1 D$ and 0.03D. $t$ denotes the age of the ice at $z^{\prime}=0$, and $z$ its depth below the surface. The initial doped signal is made from the superposition of 1200 random Gaussian peaks at decimetre scale. The upper panel shows the whole signal sequence as it evolves, resizing the axes occasionally to expand upon details. The lower panel focusses on the stretch $z^{\prime}=-5$ to $5 \mathrm{~m}$. Snapshots from the movie are shown in Fig. 10.
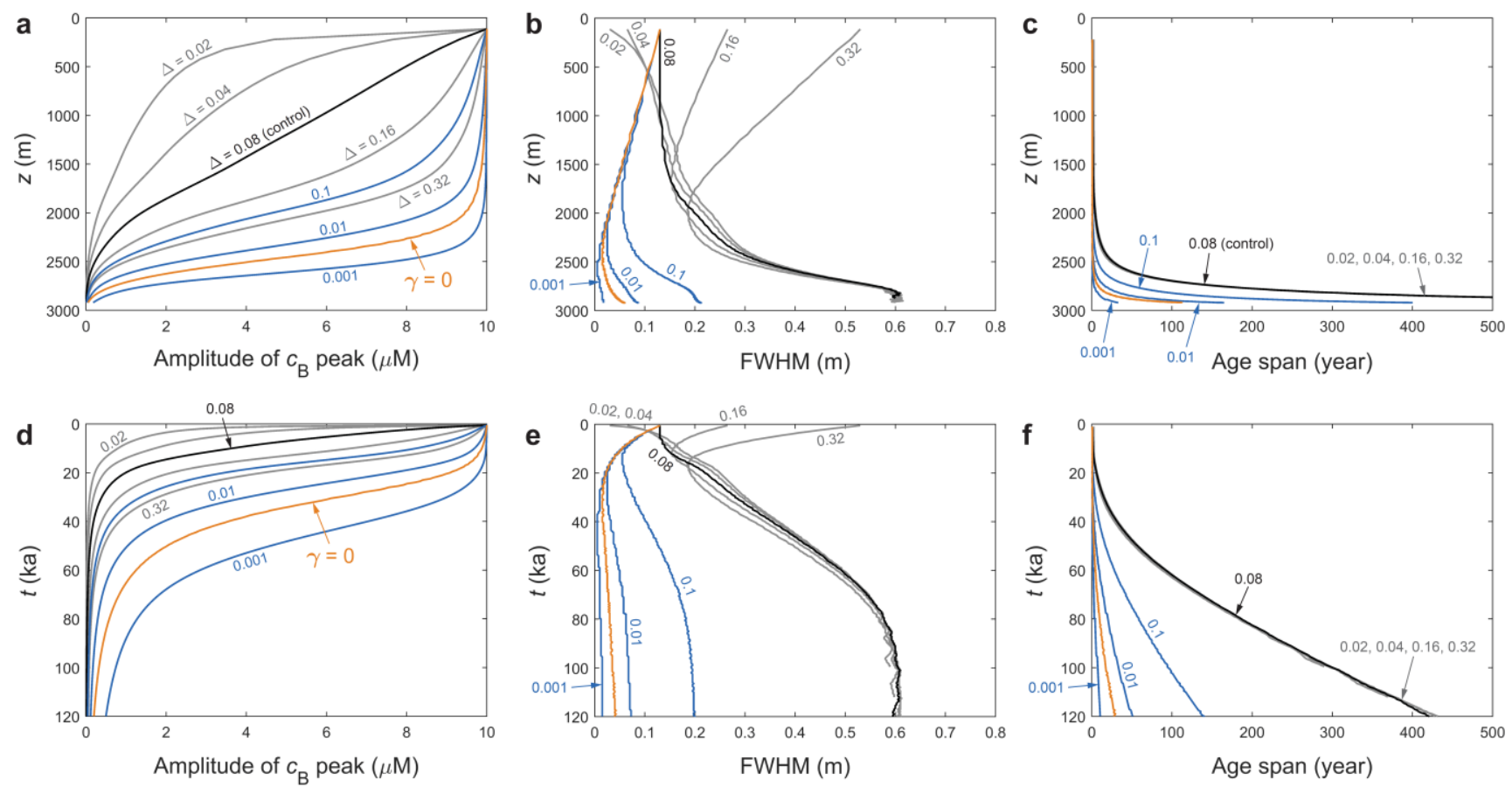

Figure S1: Changing morphometry of the signal peak - its amplitude, full width at half maximum (FWHM), age span - in the GRIP ice core for different model parameters, plotted against depth $(\mathrm{a}-\mathrm{c})$ and age of the ice (d-f). The panel organisation follows Fig. 6. The only difference from the experiments in Fig. 6 is that the doped peak here has twice the amplitude, i.e., $c_{\mathrm{B}}$ $40=1+10 \exp \left[-\left(z^{\prime} / \Delta\right)^{2}\right]$. Black curves plot the control run. Orange curves plot the run with the Gibbs-Thomson effect turned off $(\gamma=0)$. Grey curves plot the results of altering the width parameter $\Delta$ of the doped peak from 0.08 (control) to four other values. Blue curves plot the outcomes of suppressing molecular diffusivity $D$ in the control run by the multiplicative factors 0.1, 0.01 and 0.001. Parameter labels use the same colours as the curves. 

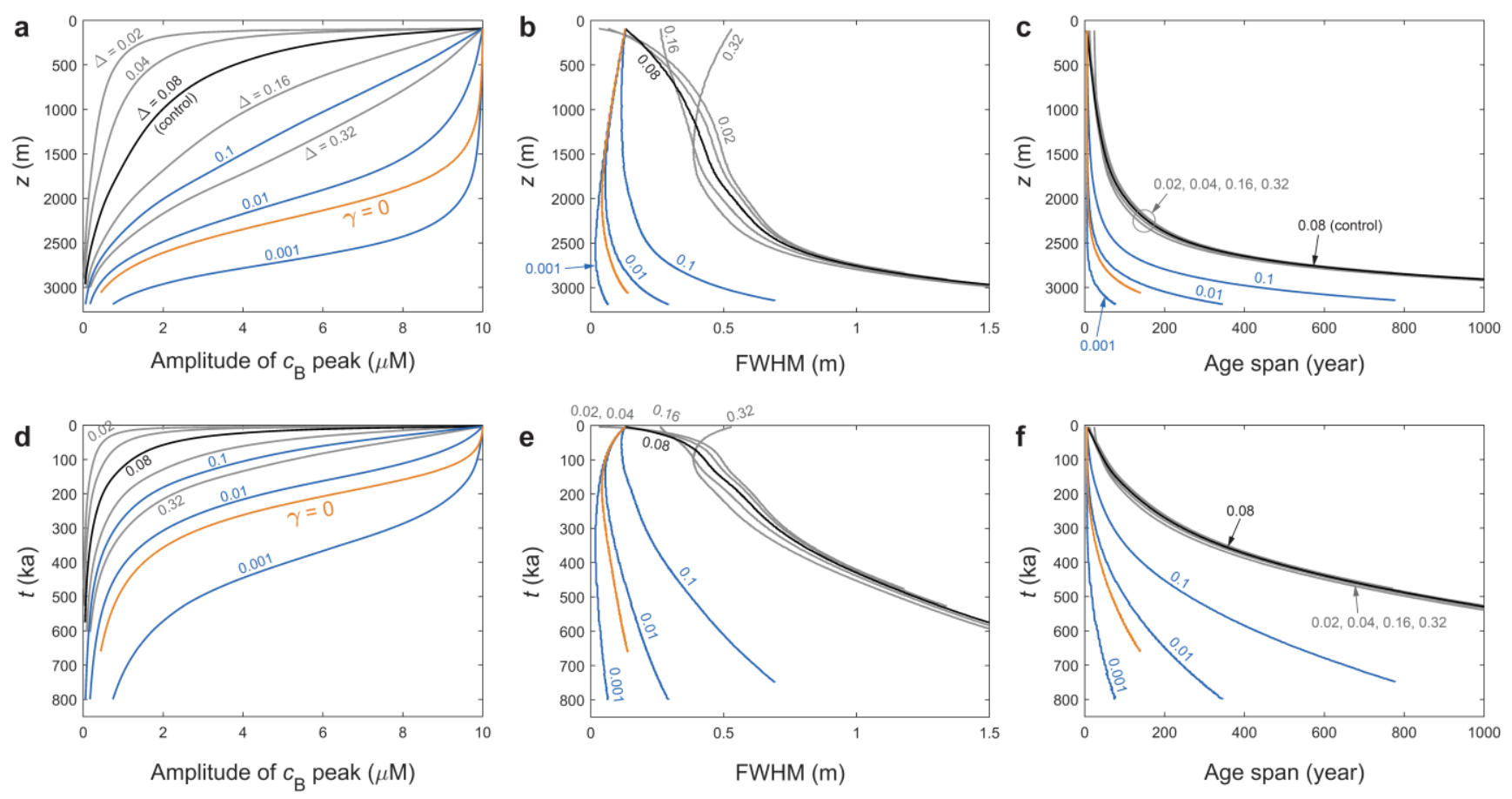

Figure S2: Changing morphometry of the signal peak - its amplitude, full width at half maximum (FWHM), age span - in the EPICA ice core for different model parameters, plotted against depth $(a-c)$ and age of the ice $(d-f)$. The panel organisation follows Fig. 7. The only difference from the experiments in Fig. 7 is that the doped peak here has twice the amplitude, i.e., $c_{\mathrm{B}}$ $50=1+10 \exp \left[-\left(z^{\prime} / \Delta\right)^{2}\right]$. See the caption of Fig. S1 for a guide to the different curves. 